\title{
Moving from MDGs to SDGs: Bangladesh Achievement and Challenges in Health Sector
}

\author{
Hossain S
}

The United Nations (UN) Millennium Development Goals (MDGs) are eight goals that all 191 UN Member States have agreed to try to achieve by the year 2015. The United Nations Millennium Declaration, signed in September 2000 commits world leaders to combat poverty, hunger, disease, illiteracy, environmental degradation, and discrimination against women.

Over the more than four decades since its independence, Bangladesh has achieved remarkable development progress, increasing its real per-capita income by more than 130 percent, reducing its poverty rate by 60 percent, and becoming well set to achieve most of the MDG targets. Bangladesh's health outcomes have been 'exceptional' in the context of its economic growth--something that the influential British medical journal The Lancet terms as a 'paradox'. The latest publication of Bangladesh MDGs Progress Report 2015, published by General Economics Division shows that Bangladesh has made noticeable progress in the areas of poverty alleviation, food security, primary school enrollment, gender parity in primary and secondary level education, lower infant and under five mortality rate, maternal mortality rate, improving immunization coverage and reducing the incidence of communicable diseases.

Bangladesh is on track in meeting the target of this goal measured in three different indicators like under-five mortality rate, infant mortality rate and immunization. The data from Bangladesh Demographic and Health Survey (BDHS) 2014 shows that there has been a remarkable decline (46 per 1,000 live births) in the under-five mortality rate since 1990 . As a consequence of this rapid rate of decline, Bangladesh has already achieved the MDG 4 target for under-five mortality (target of 48 per 1,000 live-births) by the year 2015. Similar to the under-five mortality rate, substantial reduction has been documented in the infant mortality rate (IMR) in the BDHS 2014 (NIPORT 2015) report (from 87 per 1,000 live births in 1993-94 to 38 in 2014). Recent data available from the SVRS 2013 show that the IMR is 32 per 1,000 live births in 2013 as compared with 94 in 1990. There are many successful programmes for immunization, control of diarrhea diseases and Vitamin A supplementation. Vitamin A supplementation rate was 86 per cent in 2007 and now it is more than 90 per cent. The BDHS 2014 shows that there has been a remarkable increase in the proportion of one year-old children immunized against measles which rose from 55 percent in 1993-94 to 84 percent in 2011, but has come down to 80 percent in 2014. Bangladesh is a story of success in improving maternal health. Maternal mortality declined from 322 in 2001 to 194 in 2010, a 40 per cent decline in nine years. Average annual rate of decline from 1990 has been about 3.3 per cent, while MDG requirement is 3 per cent. Bangladesh is not in an alarming position because prevalence of HIV/AIDS is currently less than 0.1 per cent which is still below an epidemic level. Prevalence of malaria per 100,000 populations was 441.5 in 2005 , which came down to 202 in 2013. Children with fever who are treated with appropriate anti-malarial drugs was 80 per cent in 2008, which was recorded at 89.5 per cent in 2013 and the target is to achieve 90 per cent in 2015 . The death rate associated with TB was 61 per 100,000, populations in 1990 and the status is 45 in 2012.

The country's huge success on fulfilling different goals of MDGs has also been recognized by the international organizations and the country has received UN award for this. As a result of the outstanding performance in improving the child immunization status, Bangladesh achieved GAVI Alliance Award in 2009 and 2012, which was given as recognition to achieving the Millennium Development Goals (MDG), particularly in reducing child mortality. The success of Bangladesh in achieving the targets of MDGs is acclaimed globally when our Hon'ble Prime Minister was awarded with 'UN MDG Awards 2010'. She was also awarded the South-South Award 'Digital Health for Digital Development' in 2011 for her innovative idea to use the Information and Communication Technology to accelerate progress of the health of women and children.

However, the attainments of a few targets of MDGs are associated with several challenges also. The existence of poverty pockets, prevalence of unemployment and underemployment among the youth, stunting and wasting

Correspondence: Dr. Md.Shahadat Hossain, Associate Professor and Head, Dept. of Physical Mesicine, Shaheed Suhrawardy Medical College, Sher-E- Bangla Nagar, Dhaka 1207, Bangladesh. 
among the under five children, reducing the dropout rate and enhancing the quality of education at the primary level, universal access to reproductive health and resource constraints are identified as stumbling blocks in fulfilling all the targets of MDGs in Bangladesh.

Yet current population challenges are not only about numbers, but also about polarization of abundance and deprivation - in other words, inequalities. An investment in adolescent girls in particular could make a significant and lasting contribution to reducing gender inequalities, help to break intergenerational cycles of deprivation and poor health, and support achievement of national priorities. On the other hand, a failure to invest in youth may reinforce inequalities and can become a major source of social instability.At the same time, the population aged 60 and older is expected to double from 2015 onward, which will need to entail the promotion of healthy ageing and economic well-being in old age as well as a supportive environment where older persons are integrated into the development process as an asset.

More to do for sustainable food security and good nutrition,one of the most critically off-track MDG targets is that of child undernutrition, which has reduced in Bangladesh in recent decades but remains a considerable concern. In terms of child health, while mortality rates have improved, major inequalities among the population segments still need to be addressed. Childhood deaths, especially by drowning, have emerged as a considerable public health concern responsible for a full quarter of the deaths among children one to four years of age.

Yet the second off-track MDG target of special concern in Bangladesh involves maternal health. Some debate exists about the statistics of the Maternal Mortality Ratio (MMR), in Bangladesh as elsewhere, although in all cases the ratio remains high; meanwhile, the Bangladesh Maternal Mortality Survey 2010 shows only 26.5 percent of births attended by skilled health personnel, with wide disparities between the richest and poorest quintiles. While there has definitely been progress overall, this debate over data suggests a critical challenge with regard to the scale and speed of improvements. Current data on the three determinants of improvement in MMR - skilled attendant at delivery, access to emergency obstetric care (EmOC) and an effective referral system to ensure women receive EmOC in case of complications - suggest that Bangladesh will have to exert far more consistent and scaled-up efforts to ensure meeting this crucial target. In addition, challenges remain in achieving universal access to reproductive health, in part because of the persistent unmet need for family planning, especially among unmarried adolescents, and as well as because of further wide regional disparities.Moving from MDGs to SDGs: Bangladesh Experience and Expectation government is taking many programmes to combat infectious diseases like TB/Malaria. Despite of taking these initiatives there is still presence of challenges. Therefore, progress on reducing the prevalence of malaria has been less impressive and needs further attention, while XDR TB (extensively drug resistant tuberculosis) is a rapidly growing issue. Epidemiological and demographic transitions also now impose a complex burden of infectious diseases alongside non-communicable diseases (NCDs), mental health, injuries and the consequences of violence. Already 21 percent of deaths among women of reproductive age in Bangladesh are due to cancer, of which cervical and breast cancers constitute nearly half. Globally, the cost of inaction in relation to NCDs - estimated in the trillions of dollars - is now recognized as a risk requiring action in all countries, and one that extends well beyond the health sector alone. While Bangladesh has posted many successes in primary health care, quality gaps and cost burdens pose a larger challenge in the post-2015 framework.

As the framework of MDGs is on the verge of its time period, world leaders are now negotiating on a more comprehensive framework of development incorporating three strands of sustainability namely social, economic and environmental. This development agenda, known as Sustainable Development Goals (SDGs) will replace the MDGs once those expire at the end of 2015.SDG's were first discussed at the UN Conference on Sustainable Development held in Rio de Janerio in June 2012 (Rio+20). In July 2014, the UN General Assembly's Open Working Group on Sustainable Development Goals presented a proposal to the Assembly containing 17 goals, 169 targets covering a broad range of sustainable development issues. This new development framework will focus on some of the burning issues like total eradication of poverty, availability and sustainable management of water and sanitation, health for all, action against climate change and sustainability of all development activities which are also very important in the context of Bangladesh.

Bangladesh has been at the forefront of the SDG's dialogues from the beginning. As one of the top performing countries of MDG's, she now looks equally confident to embrace the new goals set that are the SDG's. The SDGs include the second generation challenges of MDGs like quantity to quality shift, social growth ingredient, and thinking horizontal as well as vertical inequality. SDG is not the death of MDGs rather it is the continuum of MDGs to finish the unfinished targets of MDGs. The lessons for implementing MDGs will be very relevant in implementing the goals of SDGs

\section{References}

1. Bangladesh Demographic and Health Survey (BDHS), 2014.

2. Kent Buse and Sarah Hawkes. (2015). Health in the sustainable development goals: ready for a paradigm shift? Globalization and Health; $11: 13$.

3. World Development Indicators: Health systems; 2015. 